\title{
Advances in Stallion Semen Cryopreservation
}

\author{
Marco Antonio Alvarenga, DVM, PhD* , Frederico Ozanam Papa, DVM, PhD, \\ Carlos Ramires Neto, DVM, MS
}

\section{KEYWORDS}

- Stallion • Frozen semen • Sperm selection • Epididymal sperm • Centrifugation

- Cushion • Spermfilter

\section{KEY POINTS}

- Recent advances in extender composition have allowed increased quality and fertility of frozen stallion semen from ejaculated and epidydimal sperm.

- The use of new laboratory techniques to select and better protect sperm cells has allowed successful freezing of semen from stallions with sperm more susceptible to damage during the freezing-thawing process.

- Different quality control tests are available to better determine the quality of frozen semen. However, there is no reliable in vitro test to predict fertility.

\section{INTRODUCTION}

The use of stallion frozen semen minimizes the spread of disease, eliminates geographic barriers, and preserves the genetic material of the animal for unlimited time. Significant progress in the process of frozen thawed stallion semen and consequently fertility has been achieved over the last decade. Progress has been associated with the use of (1) new Al techniques, such as deep uterine artificial insemination (Al), which permits the use of a small number of sperm cells; (2) other cryoprotectants than glycerol and new commercially available extenders that results in better sperm cryosurvival; and (3) sperm selection techniques to increase the quality of frozen semen. New laboratory approaches are also available to evaluate and overcome the deleterious effects of cryopreservation.

These improvements not only increased fertility rates but also allowed cryopreservation of semen from "poor freezer" stallions, inducing a positive impact in the interest of different horse breed associations and owners in the use of stallion frozen semen

Department of Animal Reproduction and Veterinary Radiology, São Paulo State UniversityUNESP, Botucatu, Brazil

* Corresponding author. Department of Animal Reproduction and Veterinary Radiology, São Paulo State University-UNESP, PO BOX 560, Botucatu, São Paulo 18618970, Brazil.

E-mail address: malvarenga.fmvz.unesp.br@gmail.com 
with thousands of mares being inseminated yearly worldwide. Approximately $20 \%$ of embryo donor mares have been bred in Brazil with frozen semen and $90 \%$ of foals from Standardbred mares are produced by $\mathrm{Al}$ in Australia $^{1}$ with frozen or cooled semen.

This article reviews traditional steps and new strategies for stallion semen handling and processing, which are performed to overcome the deleterious effects of semen preservation and consequently improve frozen semen quality and fertility.

\section{PROCESSING SEMEN FOR FREEZING}

After collection, semen must be filtered to remove the gelatinous portion and debris from the ejaculate. Semen must then be diluted with skim milk base extender and evaluated for motility, viability, and sperm concentration to determine the number of straws. After this, centrifugation is performed to remove the seminal plasma and the sperm pellet is resuspended in extender containing cryoprotectant to the volume required to achieve the desired concentration. Finally, semen is loaded into straws. It is recommended after this time to perform a new analysis of motility, viability, and sperm concentration to ensure the quality and quantity of sperm placed in each straw. Next, semen is frozen using a predetermined freezing curve according to the medium to be used. The straws are stored in liquid nitrogen containers until thawing and used for insemination. It is recommended to perform another analysis of motility and sperm viability after thawing, to verify the quality of the semen to be inseminated (Fig. 1).

\section{Standard Procedures for Semen Collection}

The penis should be washed, especially the urethral fossa, with warm water to eliminate smegma and microorganisms just before collection. It is important to avoid the use of bactericidal solutions that can disrupt the normal bacterial flora from the penis.

The two most traditional methods to collect semen from a stallion involve the use of a phantom or a mare in heat. The use of phantoms is safest for the animal and operator and stallions can easily be trained to perform a collection into an artificial vagina.

There are different models of artificial vagina available to collect semen from stallions. The Missouri and Colorado models in the United States, Hannover in Europe,
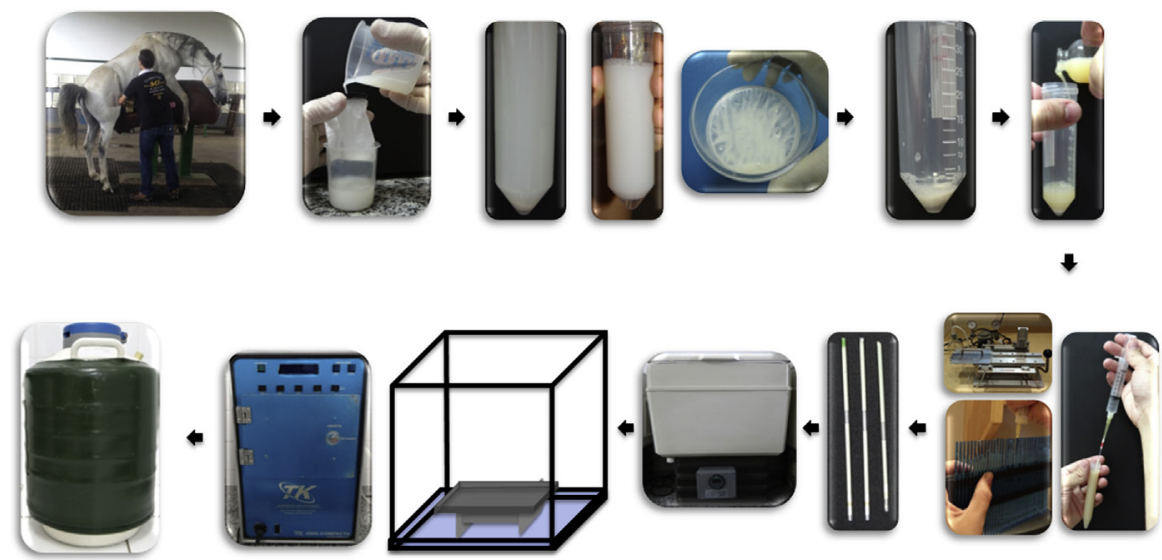

Fig. 1. Steps in the semen freezing process. First the semen is collected, filtered to remove gel, and diluted with milk-skimmed media extender. After seminal plasma is removed, the pellet of sperm is resuspended with freezing extender and finally frozen. 
and Botucatu in Brazil are the most widely used models. These models are all based on the concept of a bladder filled with hot/warm water to provide adequate pressure and temperature to stimulate stallions to ejaculate. The use of a disposable plastic inner liner is strongly recommended for sanitary reasons. Stallions can harbor potential pathogens on the penis, such as Pseudomonas, which can be transmitted to the mare during breeding. Caution should also be taken with the use of latex artificial vagina (AV) inner liners because they may be toxic when in contact with the sperm, especially when these liners are new.

The artificial vagina must be filled with hot water at around $50^{\circ} \mathrm{C}$ and may or may not be lubricated using a neutral lubricant gel. We have chosen not to use lubricants in our laboratory because many lubricants can induce damage to the sperm.

Another method to perform semen collection is with the animal on the ground. This technique is recommended for stallions with musculoskeletal problems that are unable to do a safe mount. This technique exposes the operator to greater risk and therefore must be performed with caution. The animal should be stimulated by a mare in heat properly contained and the artificial vagina should be introduced over the penis, allowing the stallion perform pelvic thrusts and ejaculate.

\section{Dilution of Semen After Collection}

The gel-free semen must be diluted with a skim milk- or casein-based extender. This dilution should be at minimum of $1: 1$ ration (vol/vol). When working with very concentrated semen, it is recommended to perform greater dilutions (2:1) to reduce damage and sperm loss during removal of seminal plasma. The diluent medium should be added to the semen and must be preheated to $37^{\circ} \mathrm{C}$ to avoid cold shock.

There is a wide variety of commercial skimmed milk- or casein-based extenders for horse semen. They provide the necessary nutrients for sperm metabolism, function as buffers to maintain the proper $\mathrm{pH}$ and osmolality control, and also protect the plasma membrane against cold shock and oxidative damage. Furthermore, because of the presence of antibiotics, these extenders play an important role in preventing bacterial growth.

\section{How to Concentrate the Sperm}

The seminal plasma is mostly produced by the accessory sex glands and expelled in fractions during ejaculation. This carries fluid and sperm, and also participates in the sperm capacitation process. ${ }^{2}$ The high volume of stallion ejaculate makes it necessary to remove them before cryopreservation.

Centrifugation is the most common technique used for concentrating sperm from a stallion ejaculate. Some studies, however, point to the damaging effects of centrifugation on the sperm: the force and duration of centrifugation required to remove the seminal plasma might negatively affect sperm motility, integrity, and recovery rate.

To remove the seminal plasma via conventional centrifugation, semen diluted with extender is placed in 50-mL conical tubes and loaded in a centrifuge. The force and time of centrifugation may negatively impact on motility, integrity, and sperm recovery rate. High centrifugal forces cause strong adhesion of the pellet, which is harmful to sperm cell, whereas low forces promote low recovery of sperm. In our experience the best combination of force and time for a good sperm recovery rate and less damage to sperm quality is $600 \times g$ for 10 minutes. $^{3}$

Immediately after centrifugation, the supernatant must be removed using a disposable catheter or needle coupled with a syringe or vacuum pump, and the pellet resuspended in the selected cryopreservation extender. If the resulting pellet is too 
compacted, the centrifugation force should be reduced in subsequent collections, or other techniques should be used to minimize excessive sperm packing.

An alternative method for removing seminal plasma from ejaculate aiming to minimizing sperm damage is the cushioned centrifugation technique. This method attempts to maximize sperm recovery from centrifuged equine semen by using high centrifugation forces, and preventing damage with a cushion fluid placed at the bottom of the centrifuge tube. ${ }^{4}$

Three commercial products are available: Eqcellsire (IMV, Lisieux, France), Cushion Fluid (Minitube, Tiefenbach,Germany), and more recently Red Cushion (Botupharma, Botucatu, Brazil). Red Cushion is a red solution that allows better visualization of the concentrated sperm pellet.

To remove the seminal plasma using cushioned centrifugation, semen must be diluted 1:1 with a skim milk-based extender and placed in a 50-mL conical tube. One to five $\mathrm{mL}$ of cushion is carefully placed in the bottom of the tube using a catheter coupled with a syringe, and centrifugation is performed at $1000 \times g$ for 20 minutes. ${ }^{5}$

Following centrifugation, the supernatant must be carefully removed via aspiration using a catheter coupled with a syringe or a vacuum pump. Next, the cushion fluid is carefully removed using a catheter, and the pellet resuspended in the desired extender (Fig. 2).

An alternative to the use of centrifugation to concentrate sperm is to filter the semen through a synthetic hydrophilic membrane (Sperm Filter, Botupharma, Botucatu, Brazil), which retains sperm and allows only the removal of seminal plasma. ${ }^{6}$ Raw semen must be diluted two parts semen and one part skim milk-based extender, placed on the filter, and using gentle motion the filter is touched against the surface of a $25-\mathrm{cm}$ Petri dish. The pore size and capillarity of the filter are such that the seminal plasma flows through, but the sperm are retained on the membrane. A predetermined volume of freezing extender is then added to the filter, and the mixture is homogenized to resuspend the sperm. The entire process lasts 5 to 10 minutes, and the same filter may be used up to 10 times for the same stallion without affecting sperm quality and recovery. The filter has also the advantage of not requiring centrifugation, which can cause mechanic damage to sperm cells (Fig. 3).

\section{Adding the Freezing Extender}

In general the extenders used for freezing semen are composed of substances to stabilize the $\mathrm{pH}$, neutralize the toxic products produced by sperm metabolism, protect

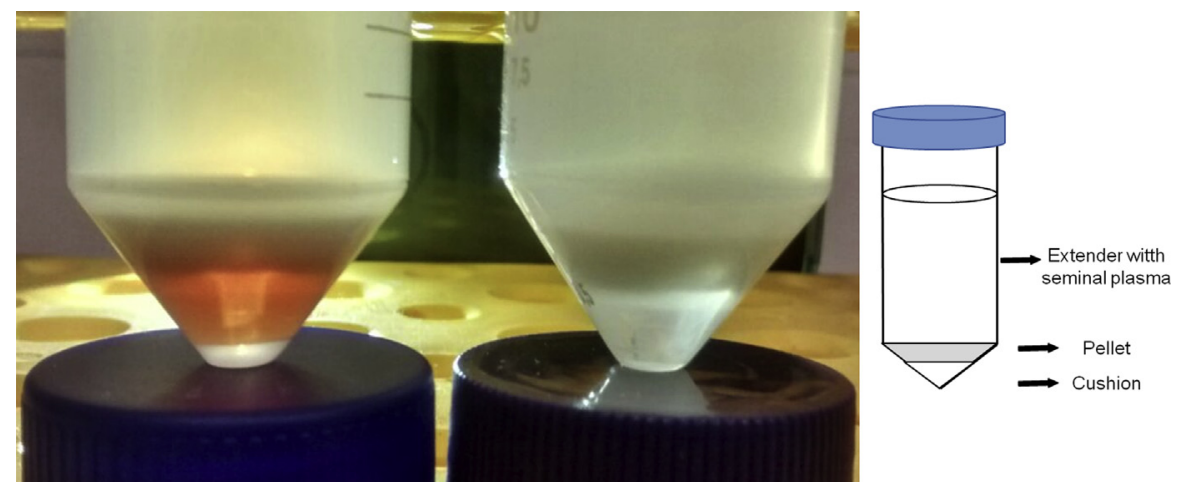

Fig. 2. Differences between the use of Red Cushion and conventional cushion fluid to centrifugate equine semen. 


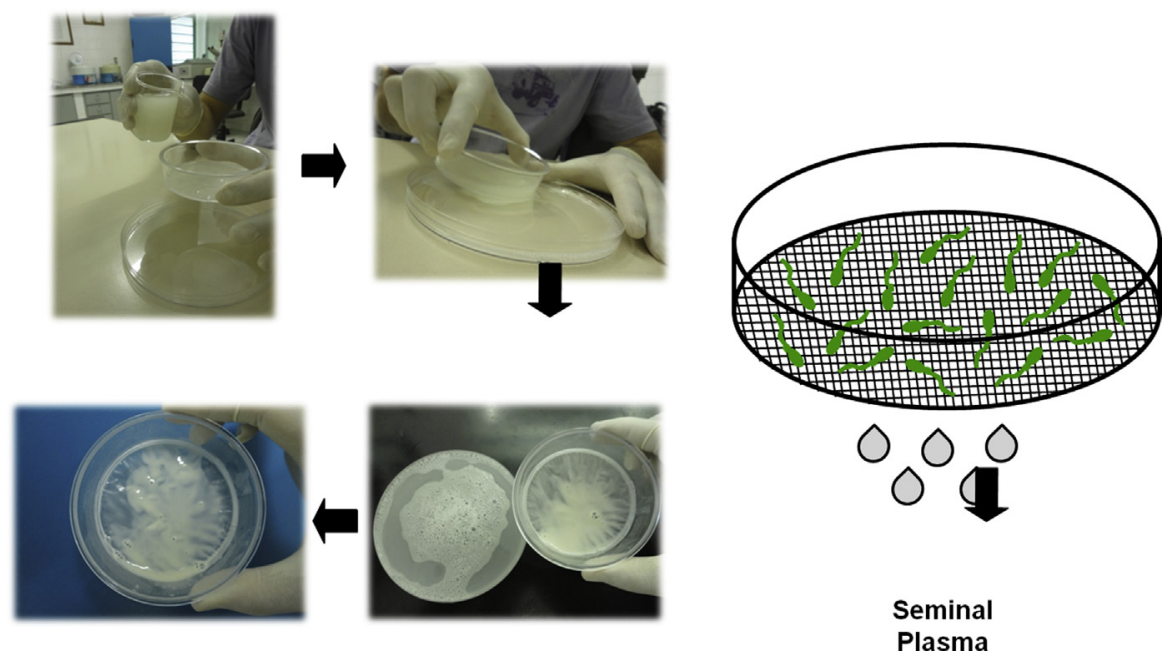

Fig. 3. Steps and schematic design for the use of Sperm Filter to remove seminal plasma.

against thermal shock, maintain the electrolytic and osmotic balance, inhibit bacterial growth, and supply energy. The extenders must also contain cryoprotectants to prevent the formation of intracellular and extracellular ice. Several commercial extenders are available, the most common being Lactose EDTA (Animal Reproduction Systems, Chino, CA), INRA freeze (IMV), and BotuCrio (Botupharma).

Several cryoprotectants have been used to freeze stallion semen. These cryoprotectants are classified as penetrating or nonpenetrating and as intracellular or extracellular. The intracellular cryoprotectants act via their ability to bind water or their colligative properties. The extracellular agents protect the sperm cells using osmotic effects to create a hypertonic environment that induces the movement of water out of the cells, dehydrating the sperm and reducing the chances for ice crystals to form inside the cells. Thus, sperm damage caused by ice formation is prevented. The nonpenetrating cryoprotectants are efficient in protecting the sperm cells during freezing without penetration. Some examples include egg yolk, milk, and some sugars.

Over the past 65 years egg yolk has been routinely used as extenders for the cryopreservation of mammalian semen and to protect sperm against thermal shock, because of the presence of low-density lipoproteins in egg yolk. These lipoproteins adhere to the cell membrane during freezing, restoring the lost phospholipids. The egg yolk seemingly induces a transient change in the composition of the phospholipids to prevent the rupture of the cell membrane and protect the sperm cells. Generally, egg yolk is used at a concentration of $20 \%$.

Sugars provide another type of nonpenetrating cryoprotection; they act on the osmotic pressure dehydrating the cells, and thus reduce the amount of intracellular water available for potential ice formation. In addition, sugars are an energy source for the sperm during incubation and protect the plasma membrane during freezing and thawing by direct interaction with the cell membrane.

Glycerol is the universal cryoprotectant used for the cryopreservation of semen. Glycerol penetrates the cell membrane by passive diffusion and remain in the membrane and cytoplasm. Although these substances cross the cell membrane until equilibrium is reached, the movement of water occurs more rapidly and causes cell dehydration. In addition to its undesirable osmotic effects, glycerol may exert direct 
action on the cell membrane, where it binds to the phospholipid head groups reducing the membrane fluidity.

Methylformamide has also been used and, in our experience, it causes less osmotic damage to sperm than glycerol because of the lower molecular weight and viscosity. ${ }^{7}$ For stallions with semen that has satisfactory freezability ("good freezers"), the use of extenders containing dimethylformamide and methylformamide may not result in a significant increase in postthaw sperm motility; however, it does increase the fertility of the frozen semen. In stallions whose semen has low resistance to cryopreservation ("bad freezers"), the use of extenders containing dimethylformamide and methylformamide provides a significant improvement in sperm motility and fertility compared with extenders containing glycerol.

The use of a combination of cryoprotectants affords better protection to sperm compared with the use of single agents. Several equine reproduction centers in Europe, the United States, and Brazil have preferentially used a commercial extender (BotuCrio), which includes a combination of methylformamide and glycerol.

The extender used in cryopreservation must be added to the semen immediately after removal of the seminal plasma. The total number of sperm in the recovered semen sample must be determined to calculate the final volume of extender required. Usually, stallion sperm are cryopreserved at a concentration between 200 and $400 \times 10^{6}$ sperm $/ \mathrm{mL}$ or 100 and $200 \times 10^{6}$ sperm per $0.5-\mathrm{mL}$ straw.

\section{Packaging}

After removal of seminal plasma and addition of the appropriated extender, the semen must be packed. Currently, semen is packaged in plastic straws with a volume of $0.5 \mathrm{~mL}$ or $0.25 \mathrm{~mL}$.

Semen is loaded into straws using automated equipment (costly) or manually. Once filled, the straws must be sealed using one of several techniques available, such as polyvinyl alcohol powder, metal and glass balls, or ultrasonic sealers. Is important to have an air bubble in the center of the straw to allow for expansion of the fluid during cryopreservation and avoid rupture of the straw during thawing.

\section{Freezing Curves}

Once the semen is packaged, the process of cooling samples to $5^{\circ} \mathrm{C}$ begins. The time required to achieve equilibrium at $5^{\circ} \mathrm{C}$ varies according to the extender used and the manufacturer's instructions. The freezing process must be slow enough to allow cell dehydration, avoiding the formation of intracellular ice crystals, and quick enough to prevent sperm exposure to supersaturated solutions as the extracellular water freezes.

The freezing curve must be performed in two steps: first the semen straws are cooled from room temperature to $5^{\circ} \mathrm{C}$ at a rate of $3^{\circ} \mathrm{C}$ to $5^{\circ} \mathrm{C}$ per minute, and then they are frozen to $-196^{\circ} \mathrm{C}$ at a rate of 20 to $50^{\circ} \mathrm{C}$ per minute.

There are particularities between extenders in the protocol to freeze stallion semen. For INRA 96, the laboratory recommends cooling for 2 hours at $5^{\circ} \mathrm{C}$ before starting the freezing process. For BotuCrio, a faster equilibration time is recommended ( 20 minutes at $5^{\circ} \mathrm{C}$ ). The recommended freezing curve after the equilibration is similar between extenders.

Two techniques are available for freezing equine semen: one uses Styrofoam coolers, and the other uses programmable semen freezer machines. Studies comparing both freezing techniques found no differences between them; however, the use of programmable systems provides better control of all procedures described previously. 
We prefer to use a 45- L Styrofoam box filled with liquid nitrogen and the straws are placed in a floating rack at $3 \mathrm{~cm}$ above the liquid nitrogen. It is important to have sufficient liquid nitrogen to keep the temperature stable for the duration of the procedure and also allow at least 3 minutes with the lid closed before placing the straws over the liquid nitrogen.

\section{Thawing and Postthaw Semen Analysis}

Several protocols are available for thawing equine semen. Some studies indicate that temperatures and times of $46^{\circ} \mathrm{C}$ for 20 seconds or $37^{\circ} \mathrm{C}$ for 1 minute are the most appropriate protocols for $0.5-\mathrm{mL}$ straws. Redilution of the semen after thawing must be avoided because it can induce osmotic damage to the cell.

Semen should be evaluated after thawing for determination of sperm motility (total and progressive), sperm morphology, vigor of sperm movement, and the number of sperm cells per straw.

For semen with acceptable postthaw motility but low fertility, we recommend the use of more sophisticated methods for semen analysis, such as analysis of the cell membrane integrity, and DNA analysis via flow cytometry. ${ }^{8}$ Acceptable parameters for postthaw semen quality are more than $50 \%$ of total motility, more than $30 \%$ of progressive motility, and expected fertility rates of $40 \%$ to $60 \%$ per cycle.

\section{Insemination Using Frozen Semen}

The ideal window of time for insemination using frozen semen is from 12 hours before to 6 hours after ovulation. Management of mares for insemination with frozen semen requires daily ultrasonographic evaluation during estrus and the induction of ovulation using human chorionic gonadotropin (1500 IU) or gonadotropin-releasing hormone (deslorelin acetate, $1 \mathrm{mg}$ ) once a follicle larger than $35 \mathrm{~mm}$ is detected in the presence of uterine edema. Twenty-four hours after inducing ovulation, ultrasound evaluation must be performed every 6 hours and insemination is performed once ovulation is detected. Alternatively, insemination is performed at fixed times at 24 and 40 hours or at 30 and 48 after induction of ovulation. In our Al protocol we routinely induce ovulation between 9 and 10 PM and expect ovulation during the day (between 32 and 48 hours after hormonal treatment), avoiding inseminations in the middle of the night.

\section{STRATEGIES TO IMPROVE SEMEN QUALITY}

Selection of stallion is performed by phenotypic assessments, such as the conformation of the animal and their athletic performance, ${ }^{9}$ unlike bovines where reproductive parameters are assessed in bulls before becoming a commercial sire. Another factor that affects sperm quality is age, and often owners decide to freeze semen from stallions that have advanced age and may be subfertile.

Several techniques have been developed to increase the sperm quality of stallions. Stallions whose semen does not resist the centrifugation process, the use of such techniques as SpermFilter, and cushioned centrifugation may be beneficial. For stallions whose semen has a low resistance to cryopreservation, the use of extenders with specific cryoprotectants, such as amines, may improve sperm cryosurvival.

When the initial quality of semen is poor, sperm selection using commercially available density gradients, such as EquiPure (Nidacon, Gothenburg, Sweden) or Androcoll-E (Minitube) can be performed before cryopreservation. ${ }^{10,11}$ These gradients select the sperm that exhibit progressive motility, cell integrity, and no morphologic defects (Fig. 4). 


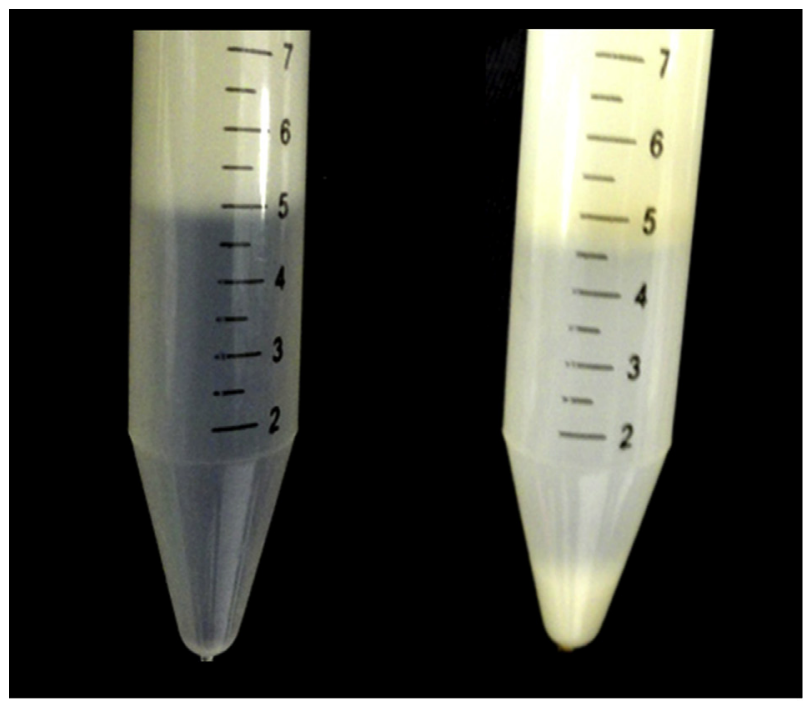

Before

After

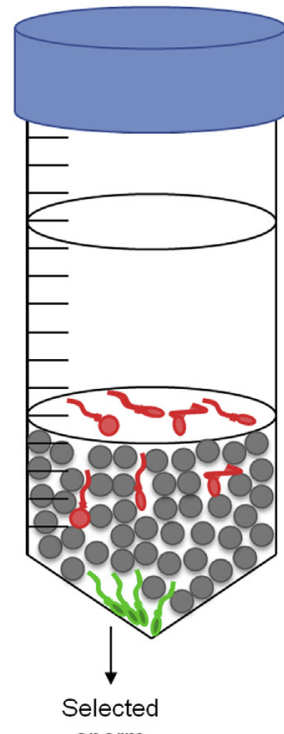

sperm

Fig. 4. Image of the semen before and after centrifugation with Equipure.

Another alternative is to select sperm after thawing. For this procedure, the contents of four straws are gently layered on top of $2 \mathrm{~mL}$ of Equipure, centrifuged at $300 \mathrm{~g}$ for 20 minutes, and the pellet resuspended with the freezing extender. Based in our experience this protocol improves the motility and the fertility of some stallions (unpublished data). It is postulated that the removal of bad quality or dead sperm that can generate oxidative reagents can improve the fertility.

\section{FREEZING OF EPIDIDYMAL SEMEN}

Epididymal sperm cryopreservation is the last chance to preserve the semen from a stallion. Following death or castration, the sperm can remain viable in the epididymis for 24 to 48 hours. In stallions exhibiting severe toxemic conditions, or when testicle removal after death was delayed, the quality of the epididymal semen, and thus the frozen semen, may be compromised.

Another interesting application for frozen epididymal sperm is the possibility to freeze semen from young stallions with no sexual experience that will be submitted to an elective orchiectomy.

The development of new extenders has allowed a significant increase in the quality and consequently fertility of stallion epididymal semen. BotuCrio has been shown to be the best extender to preserve motility and fertility of frozen epididymal sperm providing fertility rates similar to ejaculated frozen sperm. ${ }^{12}$

The epididymal sperm can also be used in such techniques as intracytoplasmatic sperm injection. Consequently, it is advised to freeze some straws with a low number of sperm per straw (eg, 5 million cells) for further use in intracytoplasmatic sperm injection.

\section{Techniques to Recover Semen from the Epididymis}

The testicles must be removed from the animal immediately after death or as soon as possible. In this case and in cases of orchiectomy, the duct deferens must be ligated 
to prevent sperm loss. The testis and attached epididymis must be rinsed with Ringer lactate or saline solution and then packaged in plastic bags or palpation gloves. When the recovery of sperm from the epididymis is not performed immediately, the testis with the epididymis can be stored at $5^{\circ} \mathrm{C}$ for 24 hours, using the same containers for cooling semen. There are two techniques to obtain sperm from the epididymis: retrograde flushing and flotation technique.

\section{The Retrograde Flushing Technique}

Retrograde flushing is the most widely used technique and involves creating pressure within the vas deferens by injecting extender until the epididymal sperm are carried by the extender through the incisions on the tail of the epididymis. The semen may be recovered by washing the tail of the epididymis with the same extender used for cryopreservation (15-20 $\mathrm{mL}$ ), or by washing with the extender used for centrifugation. We have not observed any differences on postthaw semen quality when flushing straight with the freezing extender or flushing with milk extender and then centrifuging the sample. Flushing with the freezing extender eliminates the centrifugation step. The epididymis is manually dissected to remove the fascia that surround the tail of the epididymis.

\section{Flotation Method}

In this technique, the tail of the epididymis is sliced with a scalpel blade in several small pieces to expose the epididymal sperm to the external environment. The tail of the epididymis is then placed in the freezing extender and incubated to allow the sperm to flow into the media for 15 minutes. Then, the sample is filtered using a nylon filter to remove debris. This method can also be performed after the retrograde flush technique to improve the recovery of sperm cells, especially when the epididymis is not completely dissected.

\section{Steps for Cryopreservation of Epididymal Semen}

Following the recovery of semen from the tail of the epididymis, the sperm must be concentrated using the previously discussed techniques. When the tail of the epididymis is flushed with the freezing extender, the recovered semen may be frozen directly, without centrifugation. After resuspension with the freezing extender, it is important to wait approximately 10 to 20 minutes to allow sperm to achieve the best motility parameters. Sperm motility usually improves from less than $5 \%$ to more than $70 \%$ after 10 minutes of incubation in the freezing extender. After the resuspension of the epididymal sperm, the other steps to cryopreserve are the same as described previously for ejaculated semen.

\section{REFERENCES}

1. Nath LC, McKinnon AO, Anderson GA. Reproductive efficiency of Thoroughbred and Standardbred horses in north east Victoria. Aust Vet J 2010;88:169-75.

2. Aurich JE, Kuhne A, Hoppe H, et al. Seminal plasma affects membrane integrity and motility of equine spermatozoa after cryopreservation. Theriogenology 1996; 46:791-7.

3. Dell'aqua JA, Papa FO, Alvarenga MA, et al. Effect of centrifugation an packing system on sperm parameters of equine frozen semen. Anim Reprod Sci 2001;68:324-5.

4. Loomis PR. Advanced methods for handling and preparation of stallion semen. Vet Clin Equine 2006;22:663-76.

5. Bliss SB, Voge JL, Hayden SS, et al. The impact of cushioned centrifugation protocols on semen quality of stallions. Theriogenology 2012;77:1232-9. 
6. Alvarenga MA, Papa FO, Carmo MT, et al. Methods of concentrating stallion semen. J Equine Vet Sci 2012;32:424-9.

7. Alvarenga MA, Papa FO, Landim-Alvarenga FC, et al. Amides as cryoprotectants for freezing stallion semen: a review. Anim Reprod Sci 2005;89:105-13.

8. Metcalf ES. The efficient use of equine cryopreserved semen. Theriogenology 2007;68:423-8.

9. Varner DD, Love CC, Brinsko SP, et al. Semen processing for the subfertile stallion. J Equine Vet Sci 2008;28:677-85.

10. Gutierrez-Cepeda L, Fernandez A, Crespo F, et al. Simple and economic colloidal centrifugation protocols may be incorporated into the clinical equine sperm processing procedure. Anim Reprod Sci 2011;124:85-9.

11. Morrell JM. Stallion sperm selection: past, present, and future trends. J Equine Vet Sci 2012;32:436-40.

12. Monteiro GA, Papa FO, Zahn FS, et al. Cryopreservation and fertility of ejaculated and epididymal stallion sperm. Anim Reprod Sci 2011;127:197-201. 